\section{Measurement of the Entanglement of Two Superconducting Qubits via State Tomography}

\author{
Matthias Steffen, ${ }^{*}$ M. Ansmann, Radoslaw C. Bialczak, N. Katz, Erik Lucero, R. McDermott,
} Matthew Neeley, E. M. Weig, A. N. Cleland, John M. Martinis†

Demonstration of quantum entanglement, a key resource in quantum computation arising from a nonclassical correlation of states, requires complete measurement of all states in varying bases. By using simultaneous measurement and state tomography, we demonstrated entanglement between two solid-state qubits. Single qubit operations and capacitive coupling between two superconducting phase qubits were used to generate a Bell-type state. Full two-qubit tomography yielded a density matrix showing an entangled state with fidelity up to $87 \%$. Our results demonstrate a high degree of unitary control of the system, indicating that larger implementations are within reach.

$\mathrm{T}$ he laws of quantum physics provide intriguing possibilities for a tremendous increase in computational power compared with classical computation (1). Because this power is achieved through the controlled evolution of entangled quantum states, a clear demonstration of entanglement represents a necessary step toward the construction of a scalable quantum computer $(2,3)$. However, direct demonstration of entanglement is challenging because all of the DiVincenzo criteria (4) for quantum computation must be met simultaneously. To date, only subsets of these key requirements have been demonstrated for superconducting qubits (5-9). We demonstrated all of the DiVincenzo criteria simultaneously, thus placing superconducting qubits on the road map for scalable quantum computing.

Department of Physics and California NanoSystems Institute, University of California, Santa Barbara, CA 93106, USA.

*Present address: IBM Watson Research Center, Yorktown Heights, NY 10598, USA.

†To whom correspondence should be addressed. E-mail: martinis@physics.ucsb.edu
Circuits made of superconductors and Josephson junctions are promising candidates for scalable quantum computation because of their compatibility with integrated-circuit fabrication technology (5-9). The Josephson phase qubit stands apart from other superconducting qubits because it does not use an optimal operating point. Coupling of phase qubits is thus straightforward, allowing for multiple control methods (10). With recent improvements in coherence times and amplitudes (11), and in particular the ability to measure both qubit states simultaneously (5), it is possible to use phase qubits to produce entangled states and measure them with high fidelity.

In the phase qubit circuit (Fig. 1A), the Josephson junction (with critical current $I_{0}$ ) has a superconducting phase difference, $\delta$, that serves as the quantum variable. When biased close to the critical current, the junction and its loop inductance, $L$, give a cubic potential that has qubit states $|0\rangle$ and $|1\rangle$, with an energy spacing that corresponds to a transition frequency $\omega_{10} / 2 \pi \sim 5 \mathrm{GHz}$ (Fig. 1B). This frequency can be adjusted by $\sim 30 \%$ via the bias current.
Single qubit logic operations, corresponding to rotations about the $x, y$, and $z$ axes of the Bloch sphere, were generated as follows. Rotations about the $z$ axis were produced from current pulses on the qubit bias line that adiabatically change the qubit frequency, leading to phase accumulation between the $|0\rangle$ and $|1\rangle$ states of the qubit (11). Rotations about any axis in the $x y$ plane were produced by microwave pulses resonant with the qubit transition frequency. They selectively address only the qubit energy levels, because transitions to higher-lying energy levels are off-resonance due to the anharmonicity of the potential and the shaping of the pulses (12). The phase of the microwave pulses defines the rotation axis in the $x y$ plane. The pulse duration and amplitude control the rotation angle.

The qubit state was measured by applying a strong pulse, $I_{z}$, so that only the $|1\rangle$ state tunnels out of the cubic well (Fig. 1C). Once tunneled, the state quickly decays into an external ground state that can be easily distinguished from the untunneled $|0\rangle$ state by an on-chip superconducting quantum interference device (SQUID) amplifier.

Two separate phase qubits were coupled with a fixed capacitor (5) (Fig. 1D). With the qubits labeled $\mathrm{A}$ and $\mathrm{B}$, the coupling Hamiltonian is $H_{\text {int }}=(S / 2)(|01\rangle\langle 10|+| 10\rangle\langle 01|)$, where $|01\rangle=|0\rangle_{\mathrm{A}} \otimes|1\rangle_{\mathrm{B}}$. The coupling strength, $S=$ $\left(C_{x} / C\right) \hbar \omega_{10}$, is proportional to the coupling capacitance $C_{x} \approx 3 \mathrm{fF}$, where $C \approx 1.3 \mathrm{pF}$ is the junction shunting capacitance (13) and $\hbar$ is Planck's constant $(h)$ divided by $2 \pi$. The two qubits may easily be brought into resonance, even though they are not identical, because each can be tuned over a large frequency range. On resonance, the interaction produces an oscillation with frequency $S / h$ between the states $|01\rangle$ and $i|10\rangle$; for an interaction time of $h / 4 S$, the coupling produces the gate $\sqrt{i \text { SWAP. }}$. This gate, together with single qubit gates, is universal (14). The coupling also manifests itself as an avoided level crossing of strength $S / h$ in the spectroscopy of the individual qubits (15). 
The performance of each qubit can be determined separately by strongly detuning the two qubits relative to $S / h$ so that they behave inde- pendently. A standard set of experiments, including Rabi and inversion recovery experiments, gives an energy relaxation time of $T_{1}=130 \mathrm{~ns}$

Fig. 1. Qubit circuit and experimental operation. (A) Circuit schematic for a single Josephson phase qubit, where the $X$ symbol represents the Josephson junction. The measurement is implemented with a broadband 50-ohm transmission line with cold attenuators that is connected to the flux bias line with a bias tee. (B) Operation mode of the qubit showing the potential energy, $U$, versus junction phase, $\delta$. The qubit is formed from the two lowest eigenstates $|0\rangle$ and $|1\rangle$, with a transition frequency $\omega_{10}\left(I_{\mathrm{dc}}\right) / 2 \pi=$ $5.1 \mathrm{GHz}$ that can be adjusted by varying the bias, $I_{\phi}$. (C) Measurement mode of the qubit. During the measurement

A
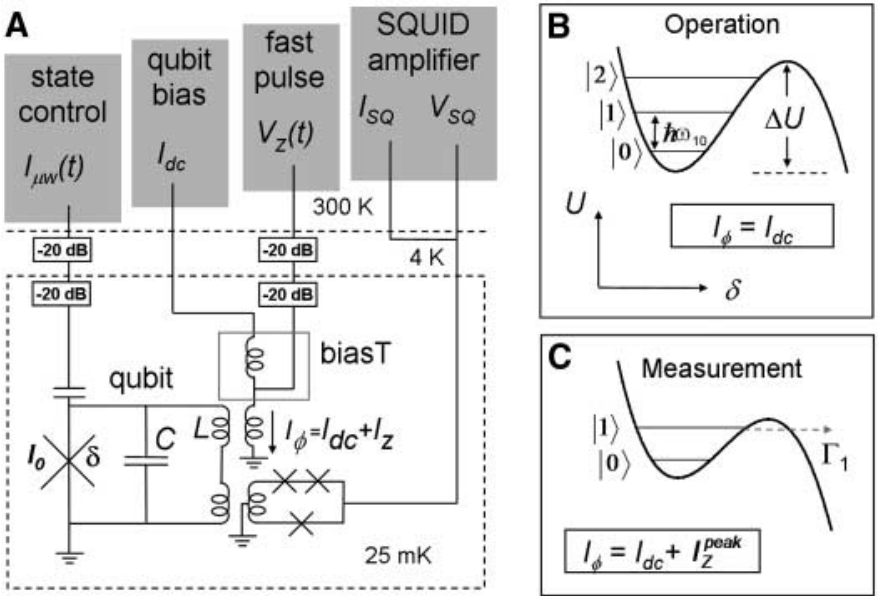

D

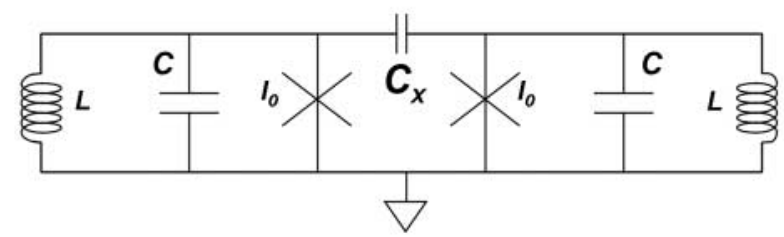

pulse, the energy barrier $\Delta U$ is lowered to increase the tunneling rate, $\Gamma$, and the tunneling probability of $|1\rangle$. (D) Circuit diagram of the coupled qubits. The loop inductance, $L$, is $\sim 850 \mathrm{pH}$, and the junction capacitance, $C$, is $\sim 1.3 \mathrm{pF}$. An interdigitated capacitor with $C_{x} \sim 3 \mathrm{fF}$ couples the qubits, giving rise to an interaction strength of magnitude $S / h=10 \mathrm{MHz}$.

Fig. 2. Coherent operations on coupled phase qubits. (A) Sequence of operations. A 10-ns-long $180_{x}$ pulse is applied to qubit $B$, populating the 01) state. After a free evolution time $t_{\text {free }}$ in which the qubits interact, the state occupation probabilities are measured by using 10-ns current pulses that induce selective tunneling of the $|1\rangle$ state. For data in (C) and (D), a 90 , and 180 zulse, respectively, is applied to qubit B after 16 ns. (B) Plot of measurement probabilities of the states $|01\rangle$, $|10\rangle$, and $|11\rangle$ as a function of $t_{\text {free }}$. Note that $P_{00}=1-P_{01}-P_{10}-$ $P_{11}$. The solid lines are the results of simulations using known measurement fidelities, relaxation times, and microwave cross talk. (C) Plot of measurement probabilities for a sequence that creates the eigenstate $\left|\psi_{2}\right\rangle=(|01\rangle-|10\rangle) / \sqrt{2}$ of the coupling Hamiltonian. After the eigenstate is formed by the $90_{z}$ pulse, it ceases to evolve with time. (D) As in (C), but with an 180 pulse. Here, the phase of the oscillation changes by 180 degrees. and a dephasing time of $T_{2}{ }^{*}=80 \mathrm{~ns}$ for each qubit. These results are consistent with measured values of an uncoupled sample (11), indicating no additional loss due to the second qubit. The measurement fidelities, defined as the probabilities of correctly identifying states $|0\rangle$ and $|1\rangle$, are $F_{0}=0.95$ and $F_{1}=0.85$, respectively.

We next tuned both qubits to $\omega_{10} / 2 \pi=5.1$ $\mathrm{GHz}$ and determined the splitting $S / h=10 \mathrm{MHz}$ by qubit spectroscopy. The time dynamics of the coupling was verified by initializing the qubits to the state $|00\rangle$ and applying to qubit $\mathrm{B}$ a $180^{\circ}$ rotation about the $x$ axis $\left(180_{x}\right.$ pulse) of 10 -ns duration. This pulse is sufficiently long to avoid unwanted transitions to other energy levels but short on the time scale of the coupling. The resulting state $|01\rangle$ is not an eigenstate of the coupling Hamiltonian and thus evolves in time according to $|\psi(t)\rangle=\cos (S t / 2 \hbar)|01\rangle-$ $i \sin (S t / 2 \hbar)|10\rangle$. After a variable free-evolution time, $t_{\text {free }}$, we simultaneously measure the state of the two qubits. Repeating the experiment about 1000 times, we determine the occupation probabilities $P_{00}, P_{01}, P_{10}$, and $P_{11}$. This sequence of operations is depicted in Fig. 2A, and the measured probabilities are plotted in Fig. 2B.

The occupation probabilities $P_{01}$ and $P_{10}$ oscillate out of phase with a period of $100 \mathrm{~ns}$, consistent with the spectroscopic measurements. The amplitude and decay of the data are also compatible with the separately measured lifetimes and measurement fidelities of the single qubits. Compared with earlier experiments (5), the amplitude of the measured oscillations is substantially larger because of improvements in single qubit fidelities. We note that the oscillations persist longer than the dephasing time, $T_{2}{ }^{*}=80 \mathrm{~ns}$, because the period of the coupled qubit oscillations (Fig. 2) is, to first order, insensitive to the detuning of the qubits. For these states, this represents a degeneracy point that is also tunable.

Although these data are consistent with the production of an entangled state at $t_{\text {free }}=25 \mathrm{~ns}$, a more stringent test includes performing coherent single qubit operations on this entangled state to verify the predicted unitary evolution of the system. After the application of a $180_{x}$ pulse on qubit $\mathrm{B}$ and a $t_{\text {free }}$ of $25 \mathrm{~ns}$, the system is in the entangled state $\left|\psi_{1}\right\rangle=(|01\rangle-i|10\rangle) / \sqrt{2}$. By then applying a $90_{z}$ pulse on qubit $\mathrm{B}$, we create the Bell state $\left|\psi_{2}\right\rangle=(|01\rangle-|10\rangle) / \sqrt{2}$. Because $\left|\psi_{2}\right\rangle$ is an eigenstate of the coupling Hamiltonian, it should not evolve with time. Implementation of this sequence of operations is complicated by the coupling interaction that occurs during the single qubit operations. Compared with the coupling interaction time, $t_{\text {free }}=25 \mathrm{~ns}$, the duration of the single qubit gates $180_{x}$ and $90_{z}$ are $10 \mathrm{~ns}$ and $4 \mathrm{~ns}$, respectively, and are thus not negligible. The excess coupled interaction during the single qubit gates can be significantly compensated by reducing the free evolution time (16) to $t_{\text {free }}=$ $16 \mathrm{~ns}$, which we checked numerically. Upon executing this sequence of operations, we verify 
A

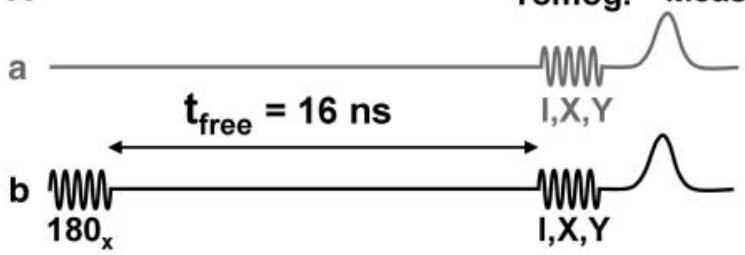

Fig. 3. State tomography of entangled qubits. (A) Sequence of operations. A $180_{x}$ pulse is first applied to qubit $B$, followed by a free evolution period of about 16 ns, creating the entangled state $\left|\psi_{1}\right\rangle=(|01\rangle-i|10\rangle) / \sqrt{2}$. State tomography is then performed by using 4-ns single qubit rotations. (B) Reconstructed $\rho_{\text {exp }}$ [real (Re) and imaginary (Im) parts] using the directly measured occupation probabilities. (C) Reconstructed density matrix $\rho_{\text {exp, } M}$ after correcting the state occupation probabilities based on the single qubit measurement fidelities. Note that matrix diagonal runs from left to right.

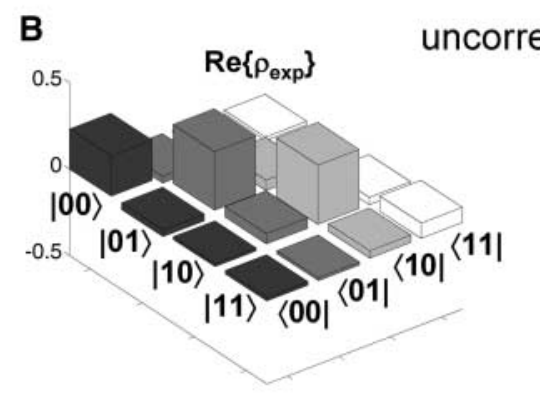

uncorrected
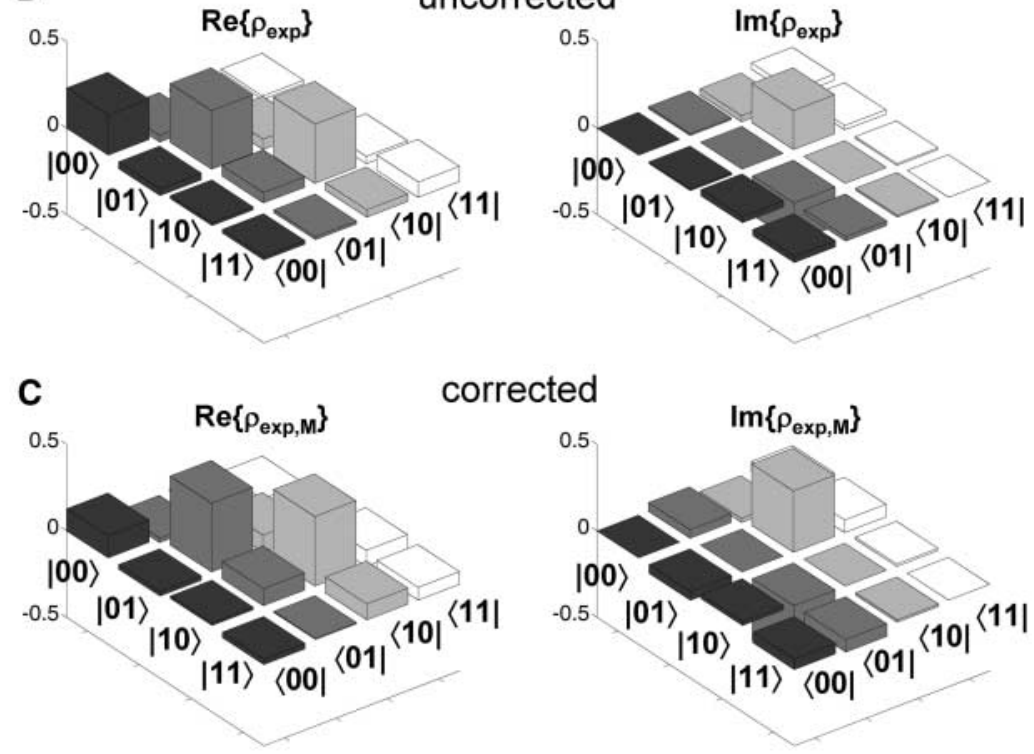

that indeed $P_{01}$ and $P_{10}$ no longer oscillate as a function of $t_{\text {free }}$ (Fig. 2C).

This observed behavior, however, could also be attributed to the destruction of coherence between the states $|01\rangle$ and $|10\rangle$ caused by the application of the $90 z$ pulse. To check this possibility, we applied a $180_{z}$ pulse on qubit B when the system is in the state $\left|\psi_{1}\right\rangle$, creating the state $\left|\psi_{3}\right\rangle=(|01\rangle+i|10\rangle) / \sqrt{2}$. Because $\left|\psi_{3}\right\rangle$ is equivalent to $\left|\psi_{1}\right\rangle$ but delayed by $t_{\text {free }}=50 \mathrm{~ns}$, a reversal of the oscillations is predicted for this experiment. This prediction is verified (Fig. 2D) and provides further evidence of an entangled state.

A full and unambiguous test of entanglement comes from state tomography $(2,3,17)$, which involves the measurement of the quantum state in all nine combinations of three measurement bases $(x,-y$, and $-z)$ for each qubit. Each measurement gives three unique probabilities (e.g., $P_{01}$, $P_{10}$, and $P_{11}$ ) for a total of 27 numbers, which are used to compute the 15 independent parameters of the unknown density matrix, $\rho$, via a least squares fit (17). The measurement basis change from $-z$ to $x$ and from $-z$ to $-y$ arises from applying a microwave pulse 90, and $90_{x}$, respectively, before measurement (11).

After calibrating the phase of the microwave pulses for the two qubits (13), we perform state tomography on $\left|\psi_{1}\right\rangle$ as indicated by the sequence of operations in Fig. 3A. As in the previous experiment, we reduced the duration of the free evolution to compensate for coupled qubit interaction during the initial $180_{x}$ pulse and the tomography pulses. After executing all nine tomography sequences and measuring the resulting occupation probabilities, we computed the density matrix, $\rho_{\text {exp }}$. The real and imaginary parts of the reconstructed $\rho_{\text {exp }}$ are shown in Fig. 3B. The imaginary off-diagonal elements $|01\rangle\langle 10|$ and $|10\rangle\langle 01|$ have nearly the same magnitude as the real diagonal components $|01\rangle\langle 01|$ and $|10\rangle\langle 10|$, revealing a coherent superposition of the states $|01\rangle$ and $|10\rangle$. This measurement unambiguously verifies that the two qubits are indeed entangled. Compared to the ideally expected density matrix, $\sigma=\left|\psi_{1}\right\rangle\left\langle\psi_{1}\right|$, we computed the fidelity of the reconstructed quantum state and find $F_{\text {exp }}=t r \sqrt{\sigma^{1 / 2} \rho_{\exp } \sigma^{1 / 2}}=0.75$

To identify the sources of fidelity loss, we first corrected for measurement error. Based on the measurement fidelities discussed earlier, we renormalized the measured occupation probabilities and calculated the intrinsic occupation probabilities (13). From this we computed a density matrix corrected for measurement, $\rho_{\text {exp }}$ (Fig. 3C), that gives an improved fidelity, $F_{\text {exp,M }}=t r \sqrt{\sigma^{1 / 2} \rho_{\text {exp,M }} \sigma^{1 / 2}}=0.87$. We attribute most of the remaining fidelity loss to singlequbit decoherence. By modeling decoherence effects (16) using the measured relaxation times, we obtained an expected $\rho_{\text {th }}$ that gives a fidelity $F_{\exp }=\operatorname{tr} \sqrt{\sigma^{1 / 2} \rho_{\text {th }} \sigma^{1 / 2}}=0.89$, which is close to the normalized measured value (18). The fact that our error is dominated by decoherence indicates good unitary control of our system and thus suggests that improvements in coherence times will directly translate to enhanced gate fidelities. Dramatic increases in coherence should be possible on the basis of straightforward improvements in the dielectric material of the shunting capacitor $(11,19)$.

Our experiments on coupled phase qubits have verified by state tomography the creation of an entangled Bell state with $87 \%$ fidelity. Given that most of the loss in fidelity can be attributed to decoherence, we believe that more complex implementations are well within reach with only modest improvements in qubit coherence times.

\section{References and Notes}

1. M. A. Nielsen, I. L. Chuang, Quantum Computation and Quantum Information (Cambridge Univ. Press, Cambridge, 2000).

2. H. Häffner et al., Nature 438, 643 (2005).

3. D. Leibfried et al., Nature 438, 639 (2005).

4. D. P. DiVincenzo, Fortschr. Phys. 48, 771 (2000).

5. R. McDermott et al., Science 307, 1299 (2005).

6. T. Yamamoto et al., Nature 425, 941 (2003).

7. D. Vion et al., Science 296, 886 (2002).

8. I. Chiorescu, Y. Nakamura, C. J. P. M. Harman, J. E. Mooij, Science 299, 1869 (2003); published online 13 February 2003 (10.1126/science.1081045).

9. A. Wallraff et al., Phys. Rev. Lett. 95, 060501 (2005).

10. M. Geller et al., personal communication.

11. M. Steffen et al., Phys. Rev. Lett. 97, 050502 (2006).

12. M. Steffen, J. M. Martinis, I. L. Chuang, Phys. Rev. B 68 , 224518 (2003)

13. Materials and methods are available as supporting material on Science Online.

14. N. Schuch, J. Siewert, Phys. Rev. A. 67, 032301 (2003).

15. A. J. Berkley et al., Science 300, 1548 (2003); published online 15 May 2003 (10.1126/science.1084528).

16. L. M. K. Vandersypen et al., Nature 414, 883 (2001).

17. L. M. K. Vandersypen et al., Appl. Phys. Lett. 76, 646 (2000).

18. A more stringent measure that quantifies the amount of entanglement, even for mixed states, is the entanglement of formation, $E(\rho)(20)$. We find $E\left(\rho_{\exp M}\right)=0.42$ compared with $E\left(\rho_{\mathrm{th}}\right)=0.61$.

19. J. M. Martinis et al., Phys. Rev. Lett. 95, 210503 (2005)

20. W. K. Wootters, Quantum Inf. Comput. 1, 27 (2001).

21. We acknowledge $S$. Waltman and the National Institute of Standards and Technology for support in building the microwave electronics. Devices were made at the UCSB and Cornell Nanofabrication Facilities, a part of the NSF-funded National Nanotechnology Infrastructure Network. N.K. acknowledges support of the Rothschild fellowship. This work was supported by Disruptive Technology Office under grant W911NF-04-1-0204 and by NSF under grant CCF-0507227. 